\title{
Generation of milling data in a virtual manufacturing framework
}

\author{
M. Matsuda
}

Faculty of Management and Informatics, Sanno Institute of

Management

1573 Kamikasuya, Isehara, Kanagawa 259-11, Japan,

Telephone:81-463-92-2211, Fax:81-463-93-0554,

E-mail:matsuda@mi.sanno.ac.jp

\section{F. Kimura}

Department of Precision Machinery Engineering, Graduate School of Engineering, The University of Tokyo

7-3-1 Hongo, Bunkyo-ku, Tokyo 113, Japan,

Telephone:81-3-3812-2111, Fax:81-3-3812-8849,

E-mail:kimura@cim.pe.u-tokyo.ac.jp

\begin{abstract}
The framework and method of implementation for the automatic generation of optimized milling data with guaranteed accuracy are discussed in this paper. By analyzing the milling data generation process, the transformation of the product description has become transparent. Namely, milling data is generated based on machining features which implicitly include a product description at the product design stage using design features. A trial milling data generation system was implemented, which can directly receive the product model with design features, convert it automatically into the required description of machining features, and produce machining data based on machining features. This trial showed that the process of milling data generation based on product description data is the same as doing the milling on the computer. Then the structure of a virtual milling system mechanism is proposed by the conceptual expansion of the implemented milling data generation system. This proposed system would generate milling data of high quality, using the product model and the milling machine model.
\end{abstract}

\section{Keywords}

product model, milling machine model, virtual manufacturing, process/operation planning, machining preparation, $\mathrm{NC}$ data generation 


\section{INTRODUCTION}

Recently, it has been said that virtual manufacturing will lead to the next generation of manufacturing systems. However, the development of new technologies are required in order to implement the entire virtual manufacturing system. Virtual manufacturing is not a simple simulation system. It is used as a tool for generation, transformation and confirmation of models or data which are required for production. In a virtual manufacturing system, various types of models are used and processed: for example the product model, physical process model and activity model. Methods for organization and processing of these models are key technologies to realize the virtual manufacturing system (Kimura, 1993a) (Kimura, 1993b). When this manufacturing concept is applied to machining preparation, the level and quality of automation in machining would be improved. That is to say, if the machining data generation system could guarantee machining results using its output data, the trial process could be omitted. This means that a large variety, small volume production, or even single product production, which do not desire machining trials, would be realized.

This paper provides the basis to the future machining system which generates machining data with guaranteed accuracy, by considering concretely a milling data generation system based on a virtual manufacturing concept. By focusing on the transformation of the product description in a milling generation mechanism on a computer, the structure of the milling data generation system becomes transparent.

\section{FLOW FOR MACHINING DATA GENERATION}

\subsection{Traditional flow}

In the traditional flow, human operators of the machining data generation system must subsequently translate design drawings into terms acceptable by computers, and input this information to get the machining data. In other words, a human operator selects the tool, extracts the corresponding shape to be machined by the tool, and then translates the product description into the desired format.

If the product designer uses a CAD (Computer Aided Design) system, human operators are still required to input some data used by the machining data generation systems including CAM (Computer Aided Manufacturing) systems. As shown in Figure 1, usually the final shape data of a product which is output from a CAD system automatically becomes the input to a CAM system. However, a CAM system requires not only shape data but also designations for the machining area, data for tools used and so on. The latter information are input by human operators who think about the facility of the factory and derive these data.

\subsection{Transformation of product description}

When a CAD system is used, the product model created at the design stage should be given as input data in the machining data generation system. The product designer usually describes the product using design features. However, most machining data generation systems accept only machining features in product descriptions. The automation of machining requires that the 
construction of a product model focuses on machining features. Here, the term "machining feature" means a volume machined by a single tool, such as a drilled hole or milled area. On the other hand, "design feature" means a unit to achieve some function, such as transmission of motion, transformation of motion and parts fixturing.

A product description created at the product design stage using design features implicitly includes machining features. However, explicit descriptions of machining features are required. In this case, the process of machining feature conversion/extraction becomes necessary. The conversion/extraction process derives explicit descriptions of machining features from descriptions of design features. Machining data which control machines and produce actual products, are generated based on these machining features. These transformations of product descriptions are shown in Figure 2.

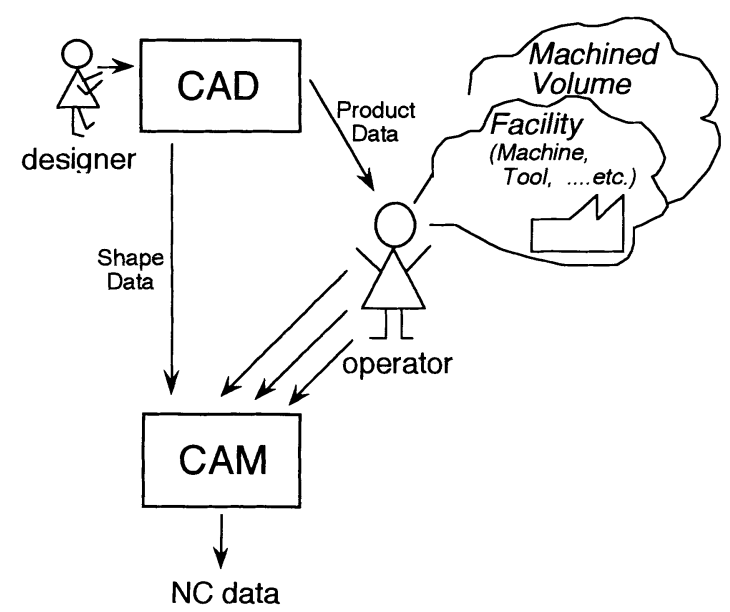

Figure 1 Traditional flow for machining data generation.

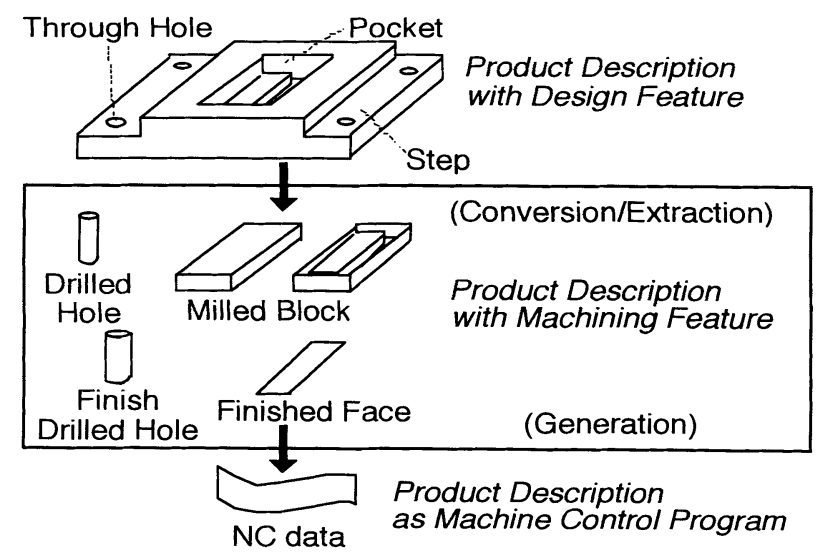

Figure 2 Transformation of product description. 


\section{MACHINING DATA GENERATION BASED ON PRODUCT MODEL}

The determination of machining features from a product model is straightforward if the form features created by a designer match the machining features recognizable by a process planner or operation planner. However, if the design features do not match the recognizable machining features, the product data include information on machining features but not explicit descriptions of them. If the machining data generation system could directly receive descriptions of the model, and convert them automatically into required descriptions, a total system of machining data generation would be possible.

Figure 3 shows the conceptual structure of the above mentioned system for machining data generation. The product model which has a description of design features is the input to this system. The system sorts the design features into two groups. One group consists of design features matching with machining features, such as a hole being produced by a drilling machine. Design features in this group are called drilled features. Drilling data is generated based on these drilled features. The other group consists of design features not matching with machining features, such as a pocket which is produced by a milling machine. Design features in this group are called milled features. A milled feature consists of free-surfaces and planes. A free-surface part is machined by ball endmill, and the part which consists of planes only is machined by a straight endmill. The system classifies composing elements of milled feature depending on surface type, and then extracts machining features by means of milled volume by one tool. Milling data is generated based on these machining features. Finally, the system merges these drilling data and milling data in order to get machining data.

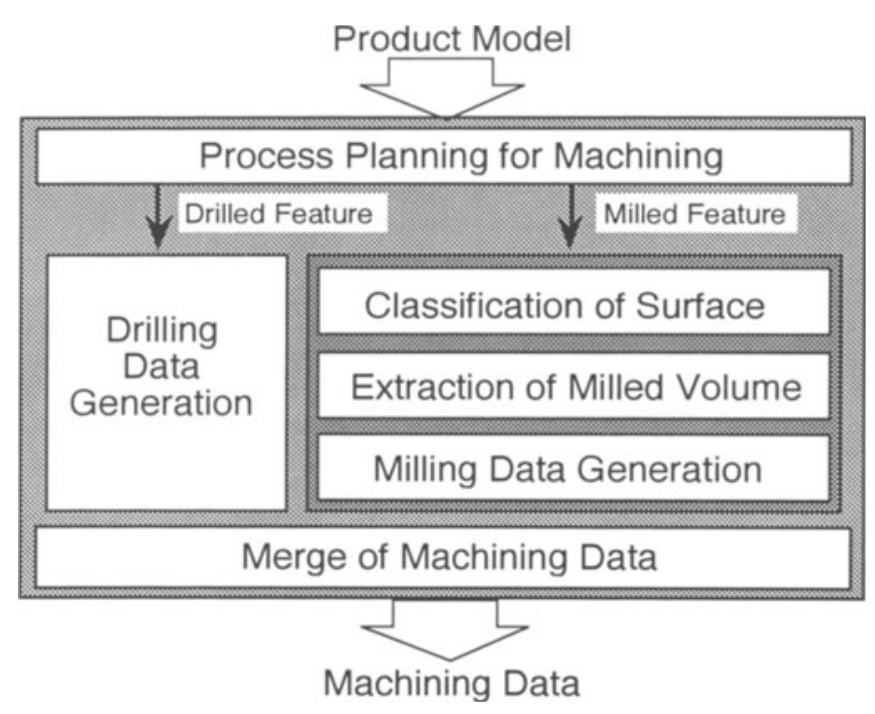

Figure 3 Conceptual structure of machining data generation system. 


\section{EXAMPLE OF MACHINING DATA GENERATION SYSTEM}

\subsection{Structure of milling data generation system}

A trial system for automatically generating milling data based on extracted milling features is developed (Matsuda, 1991). A milling feature is one type of machining feature. This system considers machining of a cavity for a die or mold. A cavity is one kind of pocket. This system treats only a pocket with a complicated $21 / 2$ dimensional shape and with round edge, chamfer edge and tapered face. A $21 / 2$ dimensional shape means the combination of several volumes which are derived by sweeping a 2 dimensional figure. This system produces a milling process plan from rough cutting to finish cutting, lays out the shape of a form tool for the finish cutting, and generates the corresponding NC data. In the rough cutting process, straight endmills are used. In order to realize high efficiency of cutting, tools with diameters as large as possible are used for rough cutting. At the intermediate cutting stage, bottom faces are finished using straight endmills. At the finish cutting stage, side faces are finished using form tools.

The structure of the milling data generation system is shown in Figure 4. This system requires form-feature descriptions of the cavity as given by product designers and information on available tools. The operation of this system proceeds through five steps. (1) The milling area generator determines cross sections at successive depths of the cavity's bottom faces. (2) The tool selector chooses tools. (3) The tool-center paths generator determines tool-center paths for each cross section and calculates milled areas. (4) The tool-center paths editor reorganizes the milled areas to derive milling features. (5) The milling process plan and NC data are generated based on extracted milling features. The methodologies for extraction of milling feature and milling data generation are explained as follows.

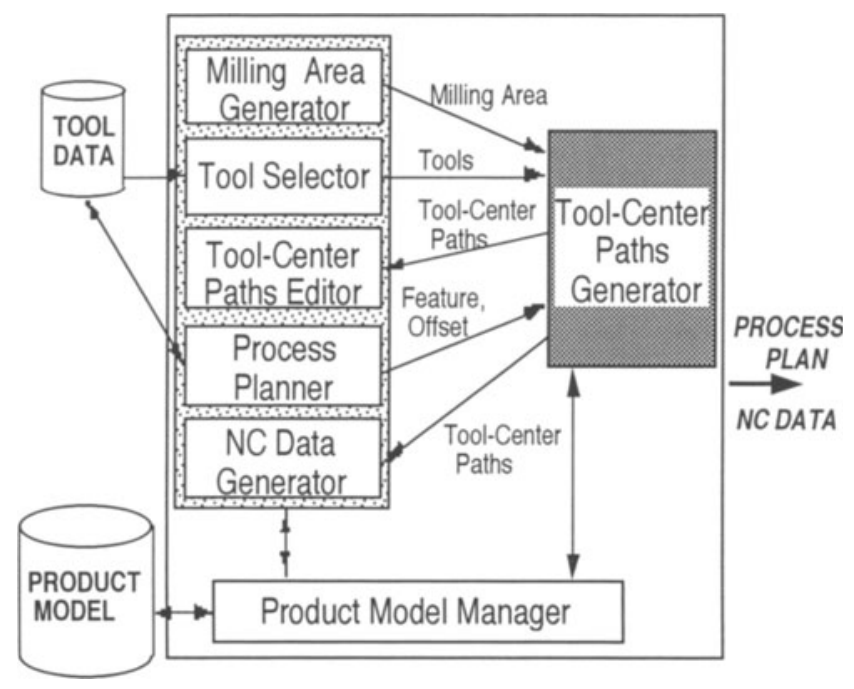

Figure 4 Structure of milling data generation system (Matsuda, 1991). 


\subsection{Computation of milled cross section at each bottom face}

A cavity is described by the shape of each bottom face, the depth of each bottom face, the inclusion relationships among bottom faces, and data for taper, round and chamfer. Bottom faces are represented using 2 dimensional B-reps solid models. The depth of each bottom face is described as an attribute of each bottom face. The inclusion relationships among bottom faces are described using two predicates: "include" and "duplicate." In Figure 5, face 2 is an island within face3. According to the top view of this figure, edge 12 of face 1 and edge 30 of face 3 are duplicated. The data for taper, round and chamfer are described as attributes of each edge.

The system processes a series of milled cross sections with the milling area at each bottom face depth. Computation among bottom face shapes to get a milled cross section depends on depth information of bottom faces and inclusion relationships among bottom faces. In Figure 5, the cavity consists of three bottom faces. At depth a, the milled cross section shows the union of face 1 and face3. At depth $b$, the milled cross section equals face3. At depth $c$, the deepest depth, the milled cross section equals the difference between face 2 and face 3 .

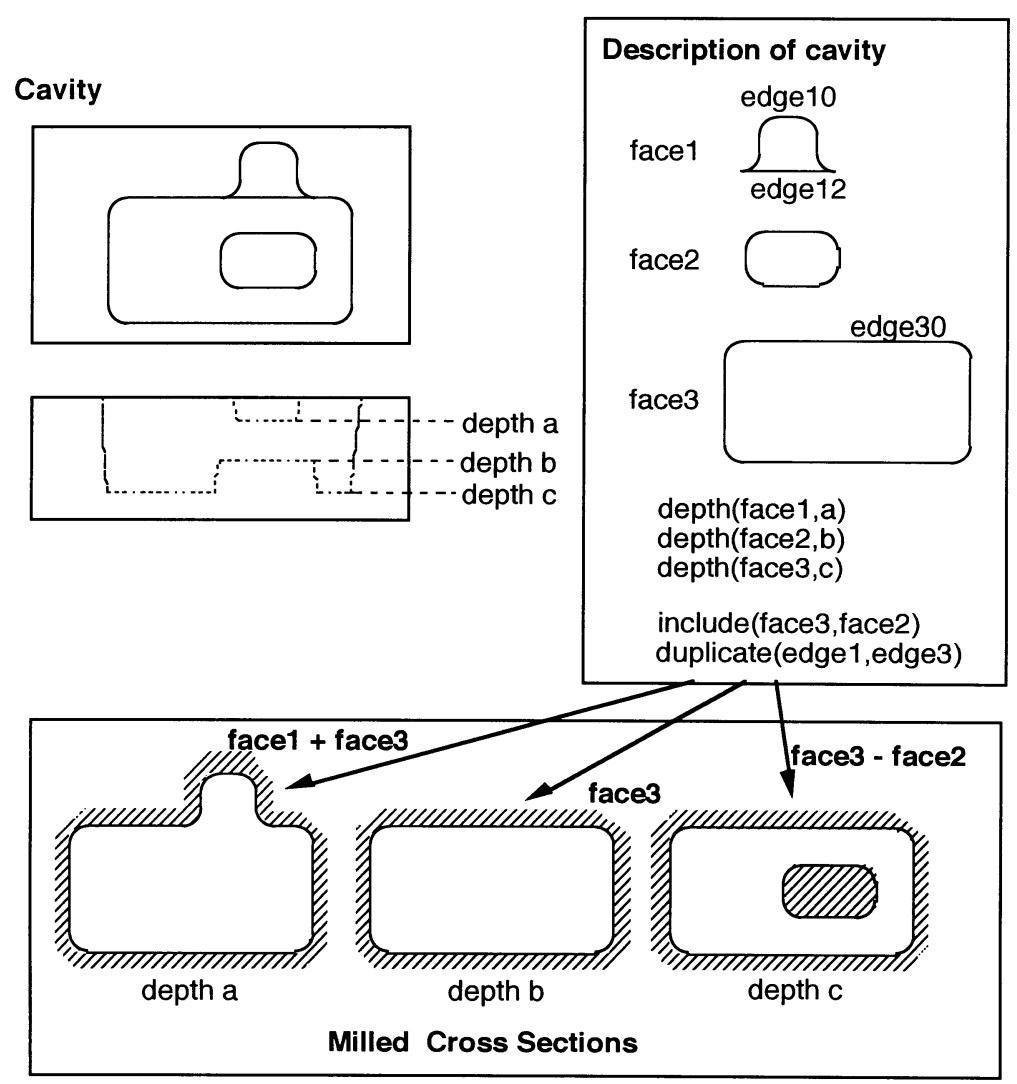

Figure 5 Computation of milled cross section. 


\subsection{Tool selection}

At this stage, each milled cross section is considered independently as a milling area. For one milling area, the selection of tool diameters of a straight endmill for rough cutting is provided depending on the corner size, distance between island areas, and the diameter of the biggest available tool. The tool diameter is selected by choosing the biggest available tool on the list with a diameter that does not exceed the distance between island areas and the corner size, and also considering the milling allowance for the next cutting stage. The final candidate list of applied tool diameters consists in an ordering of tools according to their diameters, with the biggest possible tool providing optimum milling efficiency.

\subsection{Determination by tool center path}

Figure 6 shows the tool center path determination process to get a milled area for the corresponding tool at the corresponding depth. Following a proper sequence from the list of candidate tool diameters, a bigger tool is applied for the considered milling area as follows. (1) Initial peripheral tool path center lines are generated for the cross section considered. (2) Interference points in the initial tool path center lines are determined. (3) Parts of the center line that do not interfere with other center lines are collected in order to create loops. Expansion of the tool path center line loop becomes a milled area for the corresponding tool at the corresponding depth. (4) Some tool path center line loops are rejected because the cutting areas are too small. (5) Secondary tool path center lines are generated for the remaining areas with no corresponding tool path center line loop. (6) In order to complete all loops, ends of the secondary tool center line paths are connected by generating straight lines. The system repeats the loop creation process.

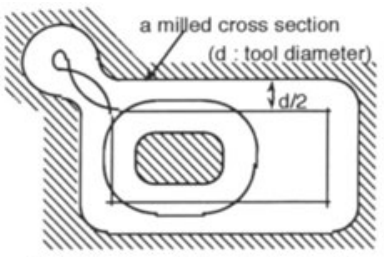

(1) Initial peripheral tool path center line generation

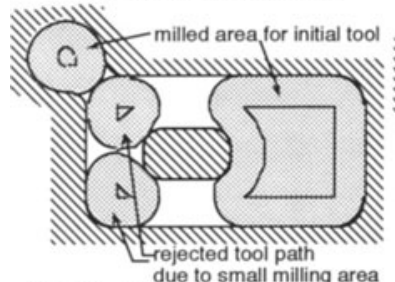

(4) Milled area and tool center line for initial tool

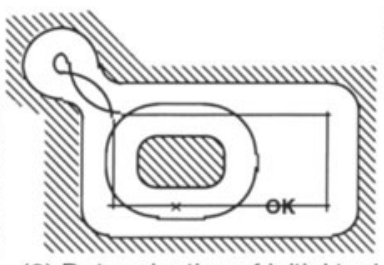

(2) Determination of initial tool path interference with part

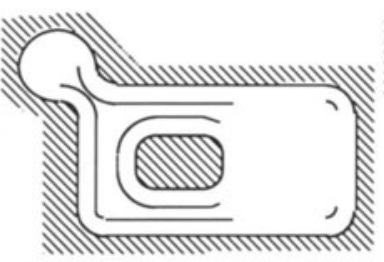

(5) Generation of secondary tool path center line

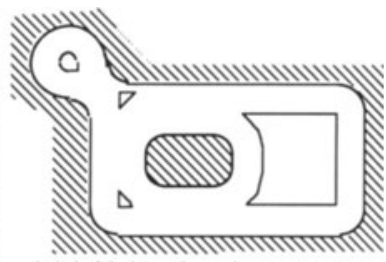

(3) Initial tool path center line selected from (2)

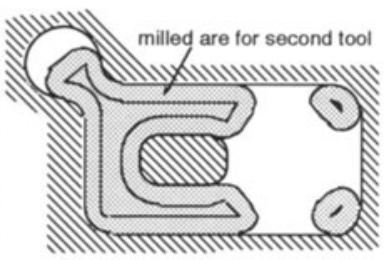

(6) Secondary tool center line path completion by system

Figure 6 Determination by tool center path 


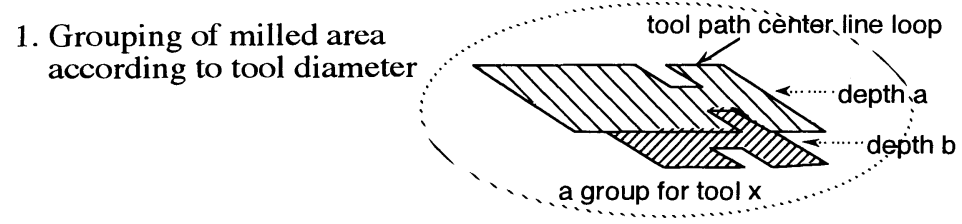

2. Division of tool center path by taper size

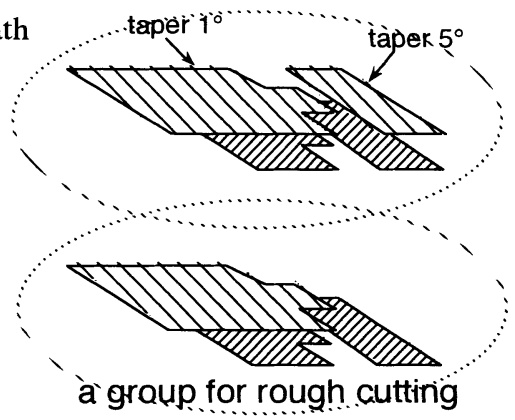

3. Difference of tool center path to eliminate duplicated part

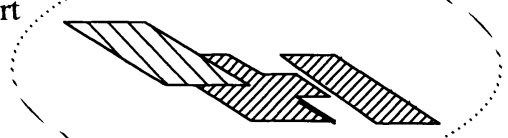

\section{a group for intermediate cutting}

4. Elimination and grouping according to original edge depth and attribute

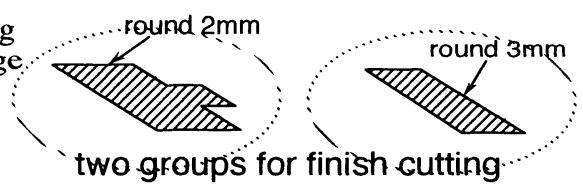

Figure 7 Editing of tool center paths.

\subsection{Editing of tool center paths}

A single endmill cuts the volume which was obtained by sweeping milled areas for one tool at a certain cutting stage. Such a volume is a milling feature. Figure 7 shows the editing of all milled areas to derive milling features. Milled areas which are provided by tool center path determination are divided into groups according to endmill diameter. These groups of tools are arranged by size, largest to smallest. In a group milled areas are arranged by depth, shallow to deep.

The process of deriving milling features for rough cutting, with a single tool of one diameter, is as follows. (1) Duplicated milled areas at each depth in one group are eliminated. (2) Tool path center line loops of milled areas are divided by the taper, round or chamfer size of the original face. Here, groups of milled areas for rough cutting are determined. One group of milled areas equals a milling feature. The process plan and NC data are obtained from these groups of milled areas. 
Milled areas in one group are determined to get milling features for intermediate cutting. If two milled areas have a duplicated part as seen from the top view, there is no bottom face on the duplicated part. In order to eliminate the duplicated part, the difference between the two milled areas is computed on the tool path center line loop.

To get groups of milled areas for finish cutting, the depth of each milled area for intermediate cutting corresponds to the depth of the edges of the original faces. If there is no equal correspondence, that milled area is eliminated. One group is divided into smaller groups by the original edge attributes of the milled area, such as "round." Form tool designs are determined depending on these small groups. One small group corresponds to a milling feature for finish cutting.

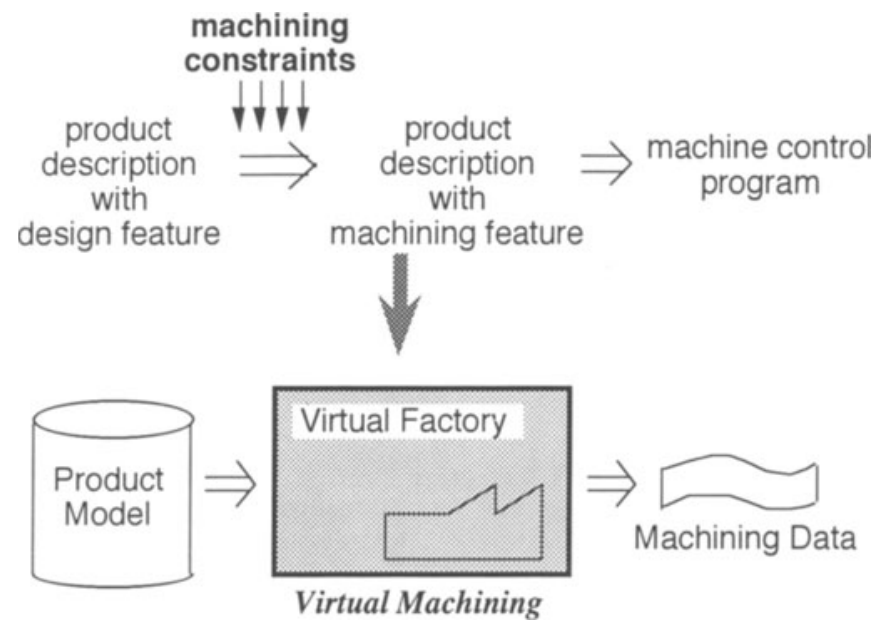

Figure 8 Concept of virtual machining (Matsuda, 1995).

\section{MILLING DATA GENERATION USING VIRTUAL MACHINE}

\subsection{Conceptual expansion to virtual machining}

In order to produce actual mechanical parts, it is necessary to generate an NC machine program based on the product model created at the design stage. The product designer usually describes the product using design features. An NC data generation system usually calculates the cutter paths based on such input data as machining features and tools. The product model created at the design stage should be provided as input data to the machining data generation system. The machining data generation system should extract machining features and plan operations such as the selection of tools, determination of cutter speeds, and calculation of cutter paths. In a machining data generation system, this means product data with design features is translated into product data with machining features. In the previous milling data generation system, it was shown that the machining data generation process is a translation process from product 
description with design feature into an NC machine program that corresponds with the machining features. Explicit descriptions of machining features are derived by applying machining constraints, such as machining methods and required tools.

These translation processes are facilitated by applying constraints on machining factors such as machining methods, machine specifications and machine functions. For example, the size of the tool diameter was the primary constraint in the previous milling data generation system. This also shows that the level of optimization and accuracy of machining data is proportionate to the number of machining constraints imposed and the accuracy of these constraints. The development of a translation system involves the implementation of mechanisms that apply machining constraints to product data. In other words, to realize this system it is necessary to build a virtual manufacturing factory within a computer and carry out machining in this virtual factory as shown in Figure 8 (Matsuda, 1995).

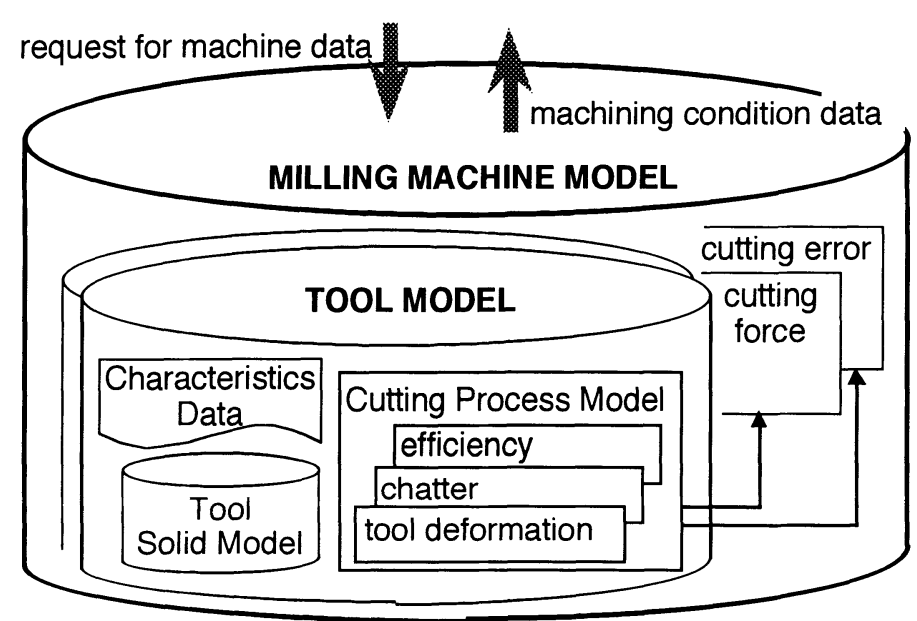

Figure 9 Virtual milling machine.

\subsection{Virtual milling machine}

A virtual machine becomes the component machine which carries out manufacturing in a virtual factory. A virtual machine is a computer model which represents the specification, function and behavior of a physical machine. A virtual machine is the controlled object in the virtual factory.

Generally, a virtual machine is composed of two models: a component model and a process model. The component model is the description of the static elements of the machine, such as machine specifications, tools, jigs or fixtures, and the controller. The process model is the description of the actual operation by means of simulation, such as cutter location, cutting force, cutting accuracy and error sensing. Each model has several variable classes. Through the combination of these classes, a flexible virtual machine modeling can be realized (Matsuda, 1995).

Figure 9 shows the virtual milling machine as a result of modeling. In this figure, the structure of a virtual milling machine is simplified, such that its components are limited to the 
tool only. A tool model consists of the solid model of the tool shape, static character data and a cutting process model by means of dynamic data which express tool working condition. Total data for milling conditions is obtained using tool models.

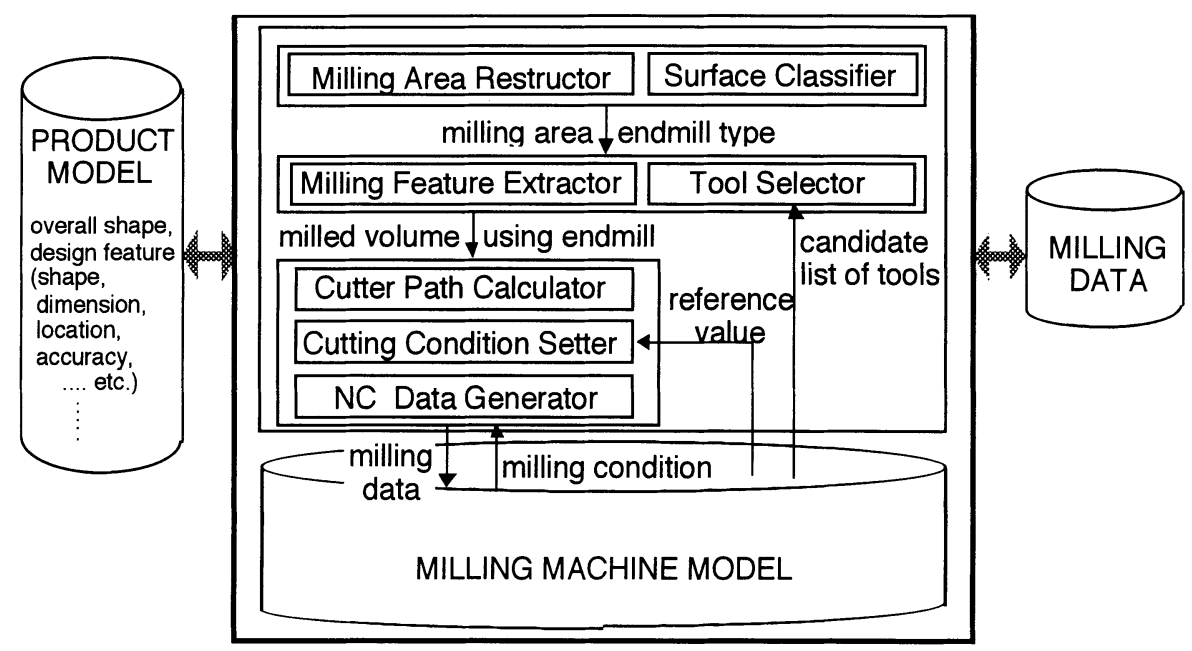

Figure 10 Structure of virtual milling system.

\subsection{Structure of virtual milling system for machining data generation}

Figure 10 proposes the structure of the virtual milling system for machining data generation. A product model is input into the system. The product model description is translated into physical machine control commands by means of usage of the virtual milling machine. A previously proposed milling machine model is used as a virtual milling machine in the system.

In the proposed system, a milled design feature is decomposed into surfaces and restructured, depending on the endmill type used. For a free-surface a ball endmill is applied. For a plane a straight endmill is used. Next, the system extracts milling features using information from the milling machine model, such as a list of available tools. Then the system calculates cutter paths, decides cutting conditions such as cutting speed, by reference to information from the milling machine model, and then generates milling data. Furthermore, the system optimizes milling data by evaluation of milling condition data which is provided by the working of the virtual milling machine based on output milling data.

\section{CONCLUSIONS}

In this paper, the milling data generation system using the product model and milling machine model is proposed. In this proposed system, the product description is transformed to milling data by applying the milling process in the computer. In other words, virtual milling is done 
using a milling machine model as a virtual machine. Input to the virtual factory is the product model which is obtained at the product design stage, and the output from the virtual factory is the NC data for milling. This milling data is optimized and guarantees the result of milling when it is used, because it is produced by virtual milling. Expanding the concept of this proposed virtual milling system leads to a general structure of a machining data generation system.

\section{REFERENCES}

Matsuda, M. and Kimura, F. (1991) Extraction of machining features for milling data generation: The 4th International Conference on Computer Application in production and Engineering, (ed. Doumeingts, G. Browne, J. and Tomljanovich, M.) Proceeding of CAPE'91 IFIP TC5, North-Holland, 353-360.

Kimura, F. (1993a) Product and Process Modeling as a Kernel for Virtual Manufacturing Environment: Annals of CIRP, 42, 147-150.

Kimura, F. (1993b) A Computer-Supported Framework for Concurrent Engineering Based on Virtual Manufacturing: Information Infrastructure System for Manufacturing, (ed. Yoshikawa, H. and Goossenaerts, J. ), IFIP WG5.3, North-Holland, 345-359.

Matsuda, M. and Kimura, F. (1995) Modeling of virtual manufacturing devices for machining data generation: The 5th International Conference on Computer Application in production and Engineering, (ed. Sun, Q. Tang, Z. and Zhang, Y.) Proceeding of CAPE'95, IFIP TC5, Chapman \& Hall, 407-414.

\section{BIOGRAPHY}

M. Matsuda is an associate professor in the Department of Computer Science of Sanno Institute of Management. She received a Dr. Eng. degree in precision machinery engineering from the University of Tokyo in 1989. She has been active in the field of CAD/CAM, process/operation planning, NC programming and numerical control. Her recent research interests include product modeling for manufacturing and virtual manufacturing. She is a member of Japan Society of Precision Engineering and Information Processing Society of Japan.

F. Kimura is a professor in the Department of Precision Machinery Engineering of the University of Tokyo. He received a Dr. Eng. Sci. degree in aeronautics from the University of Tokyo in 1974. He was a research associate at the Electrotechnical Laboratory of the Ministry of International Trade and Industry from 1974 to 1979. He then moved to the University of Tokyo, and was an associate professor from 1979 to 1987 . He has been active in the field of solid modeling, free-form surface modeling and product modeling. His research interests now include the basic theory of $\mathrm{CAD} / \mathrm{CAM}$ and $\mathrm{CIM}$, concurrent engineering, engineering simulation and virtual manufacturing. He is a member of IFIP WG5.2 and 5.3, and a member of CIRP. 